\title{
PERSISTENT LEFT SUPERIOR VENA CAVA: A DECEPTIVE ANOMALY
}

\section{Javaria Aleem, Waqas Ahmad}

Department of Radiology, Shaukat Khanum Memorial Cancer Hospital and Research Centre Lahore, Pakistan

Received: 5 October 2018 / Accepted: 18 March 2019

\begin{abstract}
Persistent left superior vena cava (SVC) is a rare but vital congenital anomaly of the thoracic venous system. It is the persistence of vessel that normally regresses during early foetal life. It has utmost importance in intervention radiology, cardiothoracic procedures and insertion of the central venous line as well as in trauma. The vessel can drain into the right atrium through the coronary sinus, directly into left atrium or through pulmonary veins. It is usually detected during routine investigations and requires surgical treatment. In our case, SVC persists on both right and left sides with enlarged coronary sinus incidentally detected during follow-up for breast cancer.
\end{abstract}

Key words: Persistent left superior vena cava, superior vena cava, vascular variant

\section{Introduction}

Persistent left superior vena cava is an infrequent anomalous systemic venous connection that poses haemodynamic and interventional challenges. It can be a misleading imaging finding in oncological investigations.

\section{Case Report}

A 55-years woman known case of right breast carcinoma underwent routine chest radiograph before starting neoadjuvant chemotherapy. On her posteroanterior chest radiograph, azygos arch shadow noted in its usual location with a focal bulge adjacent to the aortic arch [Figure 1]. She underwent contrast-enhanced computed tomography scan chest for a metastatic workup which demonstrated right superior vena cava (SVC) at normal location draining into the right atrium. Left SVC was a continuation of the left brachiocephalic vein which drains into coronary sinus posterior to the left atrium [Figures 2 and 3]. There is no communication between the left brachiocephalic vein with right SVC.

\section{Discussion}

There are two types of persistent left SVC (PLSVC) described in literature. In the majority of cases, PLSVC

Correspondence: Dr. Javiaria Aleem, Department of Radiology, Shaukat Khanum Memorial Cancer Hospital and Research Centre, Lahore, Pakistan. Email: doctor.javairia@gmail.com

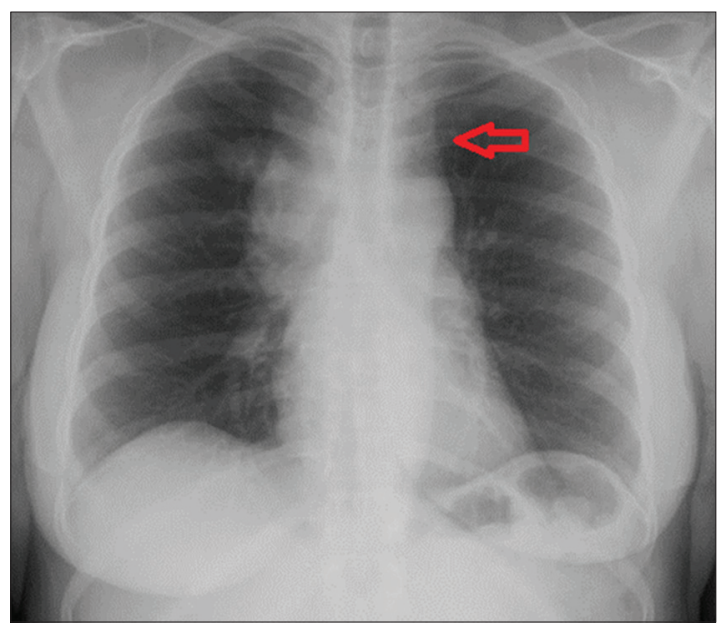

Figure 1: Chest X-ray posteroanterior view showing widening of the left paratracheal stripe by opacity due to persistent left superior vena cava (red arrow)

joins coronary sinus that drains into the left atrium. This remains clinically asymptomatic for long. In a few cases, PLSVC drain directly or indirectly through pulmonary veins into the left atrium. This creates an extracardiac right to left shunt that result in severe hemodynamic instability. This anomaly is also associated with other cardiac congenital malformations in about $40 \%$ of cases, such as atrial septal defects, ventricular septal defect, cardiac out flow tract anomalies, architectural and functional defects in conduction pathway. In such situations, right SVC is absent requiring a proper evaluation of the cardiovascular system. ${ }^{[1-3]}$ 


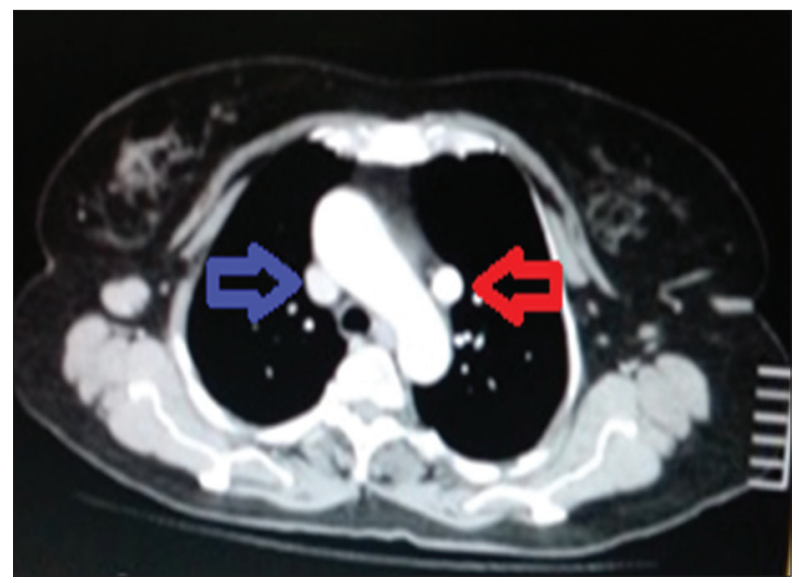

Figure 2: Axial section of post-contrast computed tomography chest showing normal anatomical right superior vena cava (SVC) (blue arrow) and variant persistent left SVC (red arrow).

PLSVC poses technical and hemodynamic difficulties during various radiological and cardiothoracic procedures. It presents difficulty in inserting catheters such as central venous catheter, Swan-Ganz catheter, permanent pacemaker or intracardiac defibrillator placement. There is a possibility of misinterpreting widening of paratracheal stripe as mediastinal lymphadenopathy during routine radiological imaging in oncology patients..$^{[4]}$ However, PLSV has important radiological and interventional implication thus should be evaluated properly and alternative access site should be considered.

\section{Conflict of Interest}

The authors declare that they have no conflict of interest.

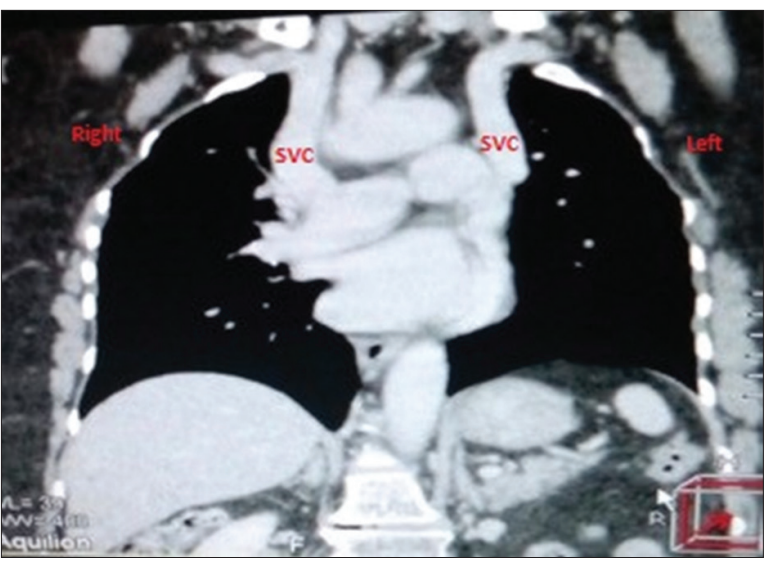

Figure 3: Coronal reformat computed tomography chest image showing normal right superior vena cava (SVC) (blue arrow) draining into the right atrium and drainage of persistent left SVC into the coronary sinus

\section{References}

1. Ratliff HL, Yousufuddin M, Lieving WR, et al. Persistent left superior vena cava: Case reports and clinical implications. Int J Cardiol 2006;113:242-6.

2. Sarodia BD, Stoller JK. Persistent left superior vena cava: Case report and literature review. Respir Care 2000;45:411-6.

3. Dearstine M, Taylor W, Kerut EK. Persistent left superior vena cava: Chest $\mathrm{x}$-ray and echocardiographic findings. Echocardiography 2000;17:453-5.

4. Goyal SK, Punnam SR, Verma G, et al. Persistent left superior vena cava: A case report and review of literature. Cardiovasc Ultrasound 2008;6:50.

\section{Authorship Contributions}

Concept and Design: JA, WA; Data Collection and interpretation: JA, WA; Literature review and writing: JA, WA; Manuscript approval: JA, WA 